\title{
CMS Web-Based Monitoring
}

\author{
William Badgett, Juan Antonio Lopez-Perez, Kaori Maeshima, Aron Soha, Balys Sulmanas, Zongru Wan.
}

\begin{abstract}
With the growth in size and complexity of High Energy Physics experiments, and the accompanying increase in the number of collaborators spread across the globe, the importance of widely relaying timely monitoring and status information has grown. To this end, we present online Web Based Monitoring solutions from the CMS experiment at CERN. The web tools developed present data to the user from many underlying heterogeneous sources, from real time messaging system to relational databases. We provide the power to combine and correlate data in both graphical and tabular formats of interest to the experimentalist, with data such as beam conditions, luminosity, trigger rates, detector conditions and many others, allowing for flexibility on the user side. We also present some examples of how this system has been used during CMS commissioning and early beam collision running at the Large Hadron Collider.
\end{abstract}

\section{INTRODUCTION}

C ERN, the European Organisation for Nuclear Research [1], is currently the world's largest particle physics laboratory. It is situated in Geneva on the border between France and Switzerland. CERN has 20 European Member States, but many non-European countries are also involved in different ways. It employs 3000 people and about 6500 visiting scientist coming from over 500 universities and research institutes from more than 80 countries. Apart from physicists, CERN's staff also includes highly specialised engineers and technicians. The research program at CERN, apart from Particle Physics in large colliders, includes projects in Nuclear Physics or in Neutrino Physics. Other active research fields are technological developments in accelerators, detectors and computer science. The Compact Muon Solenoid (CMS) [2] experiment is one of two large general-purpose particle physics detectors built on the proton-proton Large Hadron Collider (LHC) [3] at CERN. Approximately 3,600 people from 183 scientific institutes, representing 38 countries form the CMS collaboration who built and now operate the detector. Such a big collaboration requires support from a wide range of distinct monitoring and operation tools to support properly all the data taken process. Such tools must be easy to use, flexible, drillable, cooperative, resistant to security threats and available for collaborators all around the world. The whole process of data taken must be monitored efficiently, or otherwise we cannot be sure that the data obtained has enough quality as to be useful for physics analysis. If there is any problem while taking data, it should be detected (and fixed) quickly. Otherwise, important luminosity

Manuscript received May 27, 2010. Fermilab is operated by Fermi Research Alliance, LLC under Contract No. DE-AC02-07CH11359 with the United States Department of Energy.

W. Badgett, J.A. Lopez-Perez, K. Maeshima, A. Soha and B. Sulmanas are with Fermilab, IL, USA (emails: badgett@fnal.gov, juan.lopez.perez@cern.ch, maeshima@fnal.gov, soha@fnal.gov, Balys.Sulmanas@cern.ch).

Z. Wan is with Kansas State University, Manhattan, Kansas, USA (email: zrwan@fnal.gov). delivered by LHC can be lost by the CMS detector. At the time when the problems are detected, the experts who are able to fix them might be outside the experimental site so they should be able to assist and debug the problems from anywhere. Then, the ability to monitor and access the status of the CMS detector from remote locations is critical to the success of CMS.

In addition, such a big experiment is naturally a complicated system, with very different sources of information that must be monitored in different ways. Sometimes there is the need of correlating many different sources to find out and fix problems and also different CMS sub-detector might have specific needs that have to be addressed. Apart from that, there is a need for tools to support the CMS operation and run coordination, such as monitoring about efficiency, shifts performed by collaborating institutes and up-to-date and always available documentation.

It is to assess all these needs that CMS Web-Based Monitoring (WBM) [4] was proposed and developed [5].

\section{SOURCES OF INFORMATION}

\section{A. $L H C$ GMT}

The LHC General Machine Timing (GMT) system [6] provides synchronous event timing and status information over dedicated serial lines throughout the accelerator complex. The GMT signal from the LHC is available for use at CMS thorough a PC that our WBM team have installed in the CMS experimental site.

The PC has a PCI interface card that records the history of the incoming GMT events and the current status of the LHC "telegram". The telegram contains several fields indicating the current status of the LHC, including beam intensities, beam energy, safe flags, permit flags, and many others. The system can be used to provide real-time synchronous events, including changes in telegram field values and several other non-telegram accelerator events.

Our PC receives this information and converts it into a format useful for CMS, including XML, web pages, OMDS Oracle database entries and other CMS online specific formats, accessible within and outside the CMS experimental private network. This PC runs CERN's BE/CO department supplied software and a CMS specific interface to the PCI card.

\section{B. $D I P$}

LHC related data is exchanged between the LHC machine and experiments using the Data Interchange Protocol (DIP) [7]. DIP is a system which allows relatively small amounts of soft real-time data to be exchanged between very loosely coupled heterogeneous systems. These systems do not need very low latency. The data is assumed to be mostly summarised data rather than low-level parameters from the individual systems. 
Not only LHC but also all the different CERN detectors, like CMS, publish information using DIP.

That data can be accessed via software by 'subscribing' to the published trees of information. The servers publishing the information are located in different private networks (like the LHC Technical Network or the .CMS Network) so it is not trivially accessible from outside, specially from outside CERN.

Different parts of our WBM software are subscribed to these LHC and CMS DIP branches of information, to be able to monitor them, and also publish some important data. We also store in database some of that information that is relevant for the online data taking.

\section{Database}

The CMS online database contains a wealth of important information regarding the current and past states of the CMS detector. It is an oracle database, with several servers working within the private network of the experimental CMS site. Then, these data are not readily viewable from outside the CMS site due to security restrictions. A natural method to convey and display that information is to use a web server. The WBM web server was proposed to this end [5], to allow globally useful but carefully controlled access to the experimental state data. Access to the WBM server is possible both to the shift crew at the experimental site and to remote experts and users.

\section{XDAQ Flashlists}

The CMS online software (XDAQ) [8] is a software platform designed at CMS specifically for the development of distributed data acquisition systems. An important part of the real-time data taking information, like current triggers and data acquisition information, is provided using the so called XDAQ flashlists. A flashlist is a monitoring object that represents variables of the XDAQ environment that are to be monitored. Flashlist attributes are values, statuses, or texts. They refer to a set of data variables that belong to one or more qualified infospaces. They can be retrieved by XDAQ sensor software. Our WBM infrastructure looks at Trigger flashlists to monitor and display, to shifters and experts, real time trigger information very relevant to the current data taking. We also store permanently such information in database for later debugging and validation of the data taken.

\section{E. $R T L$}

The WBM Run Time Logger (RTL) [9] is a set of software tools designed to be used during experiment operation to enhance data taking efficiency. The software tools include an underlying database schema, a system to automatically log downtime occurrences and manually enter downtime reasons and finally a software for analysis of the downtime information by producing automatic data taking efficiency reports. In short, each time the CMS online system stops taking data (no matter if during a run or between different runs) the RTL infrastructure detects it, creates automatically a downtime entry, asks the shift crew for the reason of the downtime and is able to create web-based custom efficiency reports both for the shifters and for the experts, as well as for the run coordination and operation groups, with the aim of increasing the overall CMS data taking efficiency.

\section{INFRASTRUCTURE}

\section{A. WBM Core Machines \& Usage}

The WBM infrastructure includes the following 64 bits SLC5 linux [10] machines. The main usage of such machines is also briefly explained.

- lhcgmt machine: receives constantly LHC GMT and DIP information as well as other useful information like the current luminosity

- scalers machine: receives information about real-time scalers quantities like the valuable trigger rates and uses it for detecting RTL downtime entries due to stops in data taking

- SCAL Function Manager: it is controlled and managed by the central DAQ Run Control software and controls the functioning of the lhcgmt and scalers machines

- WBM web servers: currently 2 different production WBM servers are available for CMS users

- WBM development machines: these are few machines where new WBM releases are installed and tested before going into production

\section{B. Proxies \& Security}

All the machines used by the WBM infrastructure are located in the .CMS private experimental site network. For security reasons they are not directly accessible from outside that private network, with the exception of the 2 production WBM servers, which are visible from the CERN network, the so called CERN General Purpose Network (GPN). Finally, such production servers are also visible from outside CERN, worldwide, using different reverse proxies.

This system of reverse proxies is shown in fig. 1

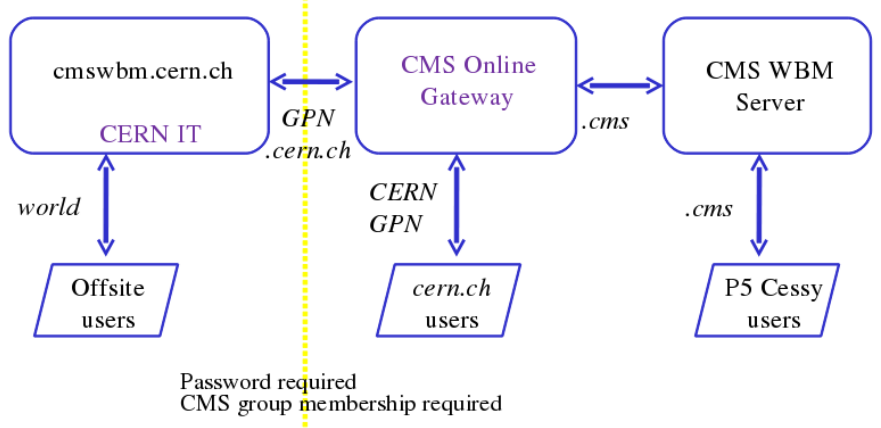

Fig. 1. WBM system of reverse proxies.

One proxy machine installed in the private CMS network and managed by the CMS system administrators allows the production WBM machines to be visible from the CERN network. Another different proxy, managed by CERN's IT department, allows these machines to be visible worldwide. Such proxy requires the standard CERN authentication, requiring CMS experiment membership and allowing very few 
exceptions, the so called CERN Single Sign On (SSO) authentication [11]. When CMS users outside CERN need to access the WBM web server, they are asked for their CERN credentials in the same way they would access any other restricted CERN web-based service. This system is integrated across all different CERN services so the authentication only needs to be performed once in the same session.

Apart from the network restrictions and the authentication system, all the different parts of the WBM web-server code have passed thorough different security reviews before the services being approved to be worldwide accessible.

\section{Code, Documentation \& Support}

WBM related infrastructure also includes the following important items:

- Documentation: there exist documentation for most of the WBM services in the CMS official Twiki sites [13] [14] as well as specific documentation in the shift leader instructions

- Twiki replica copy: a system for daily replicating important CMS online Twiki documentation into the CMS private network so if the network connection with CERN network is interrupted during some time, the documentation can still be accessed by the CMS shift crew, as the data taking should be able to afford being taking data even for any (reasonable) possible period of time with network connection problems

- Sub-version (SVN): there exist 2 different SVN [12] CERN managed code repositories for SCAL and WBM software, as well as for commissioning web pages and the software that supports the twiki replica copy system

- Savannah: a WBM specific savannah support tracking system [15] [16] [17] is available for CMS users to provide feedback, ask for new WBM features or report about possible problems

- Mailing lists: some CERN mailing lists are used for support and internal communication

\section{WBM SERVICES}

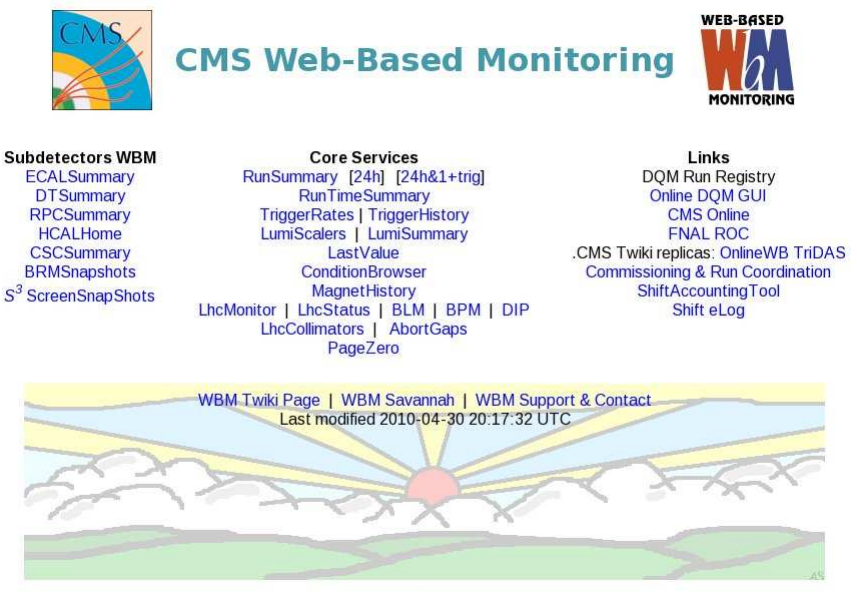

Fig. 2. Main WBM web page.
The main WBM web page [4] (see fig. 2) displays links to WBM core services in the central column, WBM sub-system services at the left and also displays links to different useful CMS online services in the right side.

\section{A. WBM Core Services}

The current list of core services is shown in the central column of the WBM web site [4]. It is documented at [14]. Some of the key services are

- RunSummary: provides access to run configuration information, with the possibility to drill down to subdetector specific displays

- L1Summary: linked from RunSummary for each specific run, it provides tabular summary of Level 1 trigger status, with drill-down options to plot rates as a function of time

- TriggerRates: produces real-time plots and tabular displays of trigger counters and rates, including physics algorithms and technical triggers

- RunTimeSummary: produces custom efficiency reports about data taking efficiency and downtime reasons

- LastValue: provides current status of the voltages and temperatures of all CMS sub-detectors, with graphical displays and drill-down details and plots

- ConditionBrowser: top-level entry point for plotting any values versus time ( $x$ vs. $t$ ), or any two time series versus each other (x vs. y)

- LhcMonitor and LhcStatus: show current LHC information and status mode

- PageZero: displays in the same page combined real-time current information from very different sources like LHC, trigger, DAQ, luminosity, magnet and CMS sub-systems status

\section{B. WBM Sub-system Services}

Many CMS sub-systems have specific tools integrated within the WBM framework, that allows them to be visible from outside the experimental site while profiting from the existing WBM infrastructure and support. The WBM framework is available for all CMS sub-systems to integrate their specific web-based software, taking into account their special needs. Current integrated CMS sub-systems tools include the ones from both the electromagnetic and the hadronic calorimeter systems and the three different muon sub-detectors. The Tracker system is in the process of being added and the infrastructure is already set up. Also the Data Quality Monitoring web tool is in the process of being integrated and it is planned to be available soon.

\section{WBM CMS Links}

Finally, many links are added for other important CMS online related sites like the electronic logbook entries or the twiki replica copies. 


\section{Usage During LHC First Collisions}

The WBM services have been proven very useful for all the different CMS online working groups during CMS commissioning and early beam collision running at the LHC. It was used in the CMS Control Room to monitor the first LHC collisions data taking and to look for problems and debug them, locally and remotely by shifters, experts and run coordination people.

One example is the display shown in figure 3 from March 30th, the first $7 \mathrm{TeV}$ collision instance. Multiple different minimum bias triggers all started showing distinct rates as the LHC proton beams collided in the CMS detector. That was used to follow the current status and to watch for possible problems at the CMS control room in the experimental site by all the shift crew and experts in and out of the CMS Control Room.

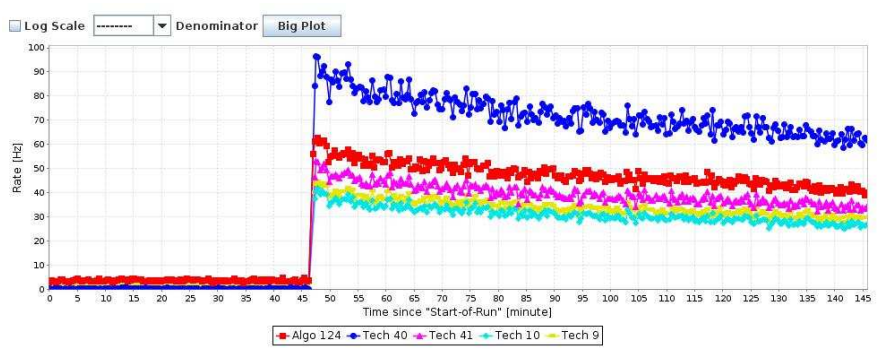

Fig. 3. WBM TriggerRates plot for first $7 \mathrm{TeV}$ collision instance.

Other examples of WBM tools used during LHC first proton physics are shown in the following figures.

In the first one (fig. 4) we show the WBM LHC Status display, giving information about current declared LHC mode, as well as previous mode and all possible modes and mode transitions. LHC proton collision physics fills are characterised by the LHC mode called Stable Beams.

In the second one (fig. 5) we show a lot of current status information during Stable Beams declared mode from many different sources (LHC, CMS DAQ, luminosity, magnet, subsystem status...).

\section{CONCLUSION}

WBM provides a central place for integrated CMS online information, to be accessed both by shift crew in charge of current data taking as well as by experts located outside CERN who may be needed for investigating possible data taking problems as well as for checking current status and efficiency. It integrates information from many different sources in a central location. It provides information about current status of the data taking, historical information for previous runs and other information like efficiency reports.

\section{ACKNOWLEDGMENT}

Many people have produced important contributions to WBM, for example Dominique Gigi in the programming of the hardware of the lhcgmt machine, Sho Maruyama in Trigger Rates cross section monitoring and Irakli Chakaberia for the Page Zero support. Also many people from the DAQ, Trigger

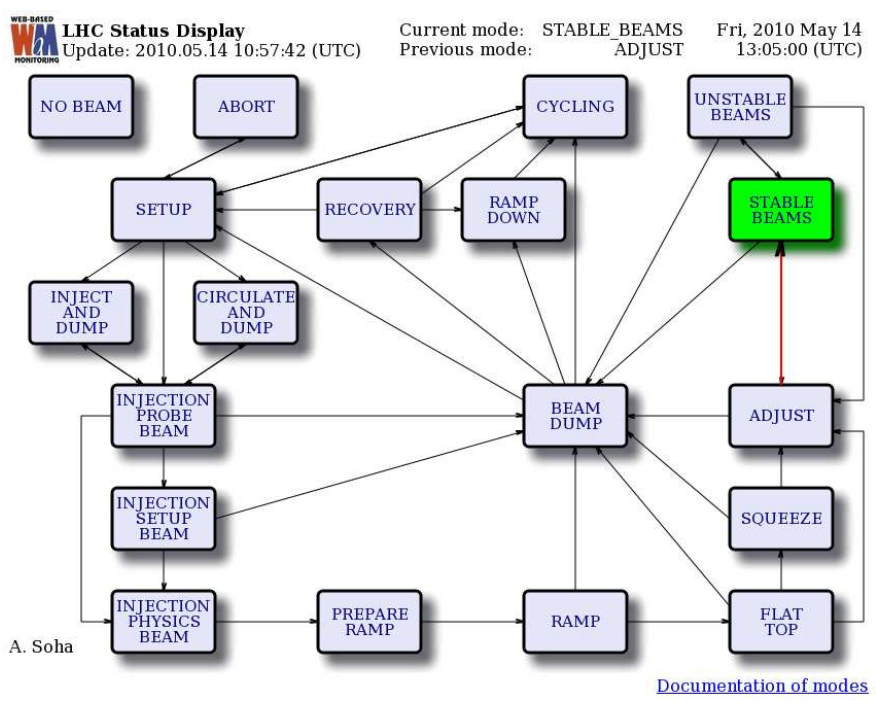

Fig. 4. WBM LHC Status Display during a real LHC proton physics fill.

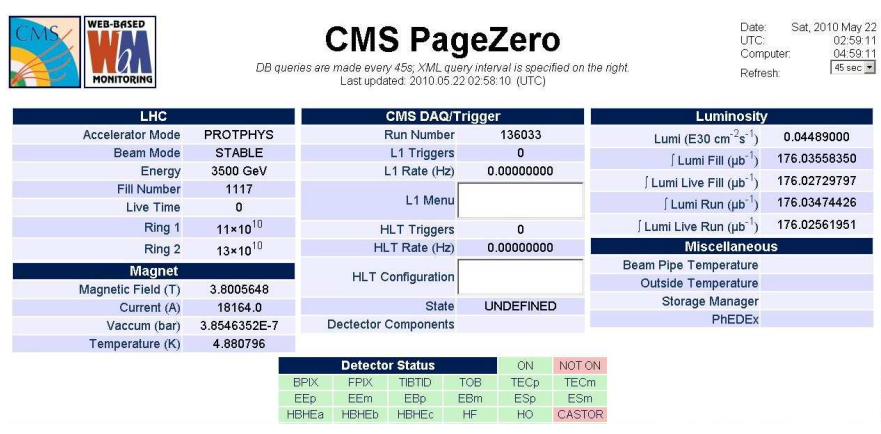

Fig. 5. WBM PageZero during a real LHC proton physics fill.

and different sub-systems groups should be thanked for their contributions, suggestions, feedback, support requests and bug reports.

\section{REFERENCES}

[1] CERN's official web site http://www.cern.ch

[2] CMS's official web site http://cms.cern.ch

[3] Large Hadron Collider's (LHC) official web site http://lhc.web.cern.ch/lhc

[4] CMS Web-Based Monitoring official public web site http://cmswbm.web.cern.ch/

[5] W. Badgett et al., "Proposal for Web Based Monitoring and Database Browsing", CMS internal note 2006/044 http://cms.cern.ch/iCMS/jsp/db_notes/noteInfo.jsp?cmsnoteid=CMS\%20IN2006/044

[6] CMS LHC General Machine Timing (GMT) documentation https://twiki.cern.ch/twiki/bin/view/CMS/CmsLhcGmt

[7] Data Interchange Protocol (DIP) web site and documentation http://lhc-commissioning.web.cern.ch/lhc-commissioning/systems/dataexchange/data-exchange.htm http://wikis.cern.ch/display/expcomm/DIP+LHC http://wikis.web.cern.ch/wikis/display/EN/DIP+and+DIM

[8] G. Bauer et al., "Monitoring the CMS data acquisition system", $2010 \mathrm{~J}$. Phys.: Conf. Ser. 219022042 http://dx.doi.org/10.1088/1742-6596/219/2/022042 CMS Online Software project (XDAQ) official web site https://svnweb.cern.ch/trac/cmsos XDAQ flashlists documentation https://twiki.cern.ch/twiki/bin/view/XdaqWiki/XMAS/Flashlists 
[9] W. Badgett et al., "CMS Runtime Logger", CMS internal note 2009/016 $\mathrm{http} / / / \mathrm{cms}$.cern.ch/iCMS/jsp/db_notes/noteInfo.jsp?cmsnoteid=CMS\%20IN$2009 / 016$

[10] Scientific Linux CERN official web site http://linux.web.cern.ch/linux

[11] CERN SSO authentication official web site https://cern.ch/authentication

[12] Sub-Version (SVN) revision control system official web site http://subversion.apache.org/

[13] WBM documentation Twiki pages https://twiki.cern.ch/twiki/bin/view/CMS/OnlineWBWebBasedMonitoring https://twiki.cern.ch/twiki/bin/view/CMS/WebBasedMonitoringServices https://twiki.cern.ch/twiki/bin/view/CMS/WebBasedMonitoringTutorial

[14] Twiki documentation tool official web site http://www.twiki.org/

[15] Official WBM savannah web site https://savannah.cern.ch/projects/wbm/

[16] Official CERN specific savannah web site https://savannah.cern.ch/

[17] Official savannah software web site http://gna.org/projects/savane/ 